\title{
EXPONENTIAL RATE OF ALMOST-SURE CONVERGENCE OF INTRINSIC MARTINGALES IN SUPERCRITICAL BRANCHING RANDOM WALKS
}

\author{
ALEXANDER IKSANOV, ${ }^{*}$ National Taras Shevchenko University of Kyiv \\ MATTHIAS MEINERS, ${ }^{* *}$ Universität Münster
}

\begin{abstract}
We provide sufficient conditions which ensure that the intrinsic martingale in the supercritical branching random walk converges exponentially fast to its limit. We include in particular the case of Galton-Watson processes so that our results can be seen as a generalization of a result given in the classical treatise by Asmussen and Hering (1983). As an auxiliary tool, we prove ultimate versions of two results concerning the exponential renewal measures which may be of interest in themselves and which correct, generalize, and simplify some earlier works.
\end{abstract}

Keywords: Branching random walk; martingale; rate of convergence; renewal theory

2010 Mathematics Subject Classification: Primary 60J80

Secondary 60K05; 60G42

\section{Introduction and main result}

The Galton-Watson process is the oldest and probably best understood branching process in probability theory. There is a vast literature on different aspects of these processes ranging from simple distributional properties to highly nontrivial results on convergence in function spaces. In particular, in [3, Section II.3-4], Asmussen and Hering investigated the rate of the almost-sure convergence of the normalized supercritical Galton-Watson process to its limit and, among other things, pointed out a criterion for the exponential rate of convergence (see [3, Theorem 4.1(i)]). The aim of the present paper is to prove a counterpart of this last result for branching random walks (BRWs), which form a generalization of the Galton-Watson processes.

We proceed with a formal definition of the BRW. Consider an individual, the ancestor, which we identify with the empty tuple $\varnothing$, located at the origin of the real line at time $n=0$. At time $n=1$ the ancestor produces a random number $J$ of offspring which are placed at points along the real line according to a random point process $\mathcal{M}=\sum_{i=1}^{J} \delta_{X_{i}}$ on $\mathbb{R}$ (particularly, $J=\mathcal{M}(\mathbb{R})$ ). We enumerate the ancestor's children by $1,2, \ldots, J$ (note that we do not exclude the case that $J=\infty$ with positive probability). The offspring of the ancestor form the first generation. The population further evolves following the subsequently explained rules. An individual $u=u_{1} \cdots u_{n}$ of the $n$th generation with position $S(u)$ on the real line produces at time $n+1$ a random number $J(u)$ of offspring which are placed at random locations on $\mathbb{R}$ given

Received 26 October 2009; revision received 10 February 2010.

* Postal address: Faculty of Cybernetics, National Taras Shevchenko University of Kyiv, 01033 Kiev, Ukraine.

Email address: iksan@unicyb.kiev.ua.

This work was commenced while A. Iksanov was visiting Münster in January 2009. A. Iksanov thanks G. Alsmeyer, M. Meiners, and Institut für Mathematische Statistik for invitation, hospitality, and financial support.

** Current address: Department of Mathematics, Uppsala University, 75106 Uppsala, Sweden. 
by the points of the random point process

$$
\delta_{S(u)} * \mathcal{M}(u)=\sum_{i=1}^{J(u)} \delta_{S(u)+X_{i}(u)}
$$

where $\mathcal{M}(u)=\sum_{i=1}^{J(u)} \delta_{X_{i}(u)}$ denotes a copy of $\mathcal{M}$ (and $J(u)=\mathcal{M}(u)(\mathbb{R})$ ). The offspring of individual $u$ are enumerated by $u 1=u_{1} \cdots u_{n} 1, \ldots, u J(u)=u_{1} \cdots u_{n} J(u)$, the positions of the offspring are denoted by $S(u i), i=1, \ldots, J(u)$, and the set of all possible individuals is denoted by $\mathbb{V}$, i.e. $\mathbb{V}=\bigcup_{n>0} \mathbb{N}^{n}$. It remains to state that $(\mathcal{M}(u))_{u \in \mathbb{V}}$ is assumed to be a family of independent and identically distributed (i.i.d.) point processes. Note that this assumption does not imply anything about the dependence structure of the random variables $X_{1}(u), \ldots, X_{J(u)}(u)$ for fixed $u$. The point process of the positions of the $n$th generation individuals will be denoted by $\mathcal{M}_{n}$. The sequence of point processes $\left(\mathcal{M}_{n}\right)_{n \in \mathbb{N}_{0}}$ is then called a branching random walk. Throughout the paper, we assume that $\mathrm{E} J>1$ (supercriticality), which means that the population survives with positive probability. Note that, provided that $J<\infty$ almost surely (a.s.), the sequence of generation sizes in the BRW forms a GaltonWatson process.

An important tool in the analysis of the BRW is the Laplace transform of the intensity measure $\xi:=\mathrm{E} \mathcal{M}$ of $\mathcal{M}$,

$$
m:[0, \infty) \rightarrow[0, \infty], \quad \theta \mapsto \int_{\mathbb{R}} \mathrm{e}^{-\theta x} \xi(\mathrm{d} x)=\mathrm{E} \int_{\mathbb{R}} \mathrm{e}^{-\theta x} \mathcal{M}(\mathrm{d} x) .
$$

We define $\mathfrak{D}(m):=\{\theta \geq 0: m(\theta)<\infty\}$, and, as a standing assumption, we assume the existence of some $\gamma>0$ such that $m(\gamma)<\infty$ (equivalently, $\mathfrak{D}(m) \neq \varnothing$ ). Possibly after the transformation $X_{i} \mapsto \gamma X_{i}+\log m(\gamma)$, it is no loss of generality to assume that $\gamma=1$ and

$$
m(1)=\mathrm{E} \int_{\mathbb{R}} \mathrm{e}^{-x} \mathcal{M}(\mathrm{d} x)=\mathrm{E} \sum_{i=1}^{J} \mathrm{e}^{-X_{i}}=1 .
$$

Set $Y_{u}:=\mathrm{e}^{-S(u)}$ and

$$
\bar{\Sigma}_{n}:=\mathrm{E} \sum_{|u|=n} Y_{u} \delta_{S(u)}, \quad n \in \mathbb{N},
$$

where $\sum_{|u|=n}$ denotes the summation over the individuals of the $n$th generation, and let $\left(S_{n}\right)_{n \in \mathbb{N}_{0}}$ denote a zero-delayed random walk with increment distribution $\bar{\Sigma}_{1}$. We call $\left(S_{n}\right)_{n \in \mathbb{N}_{0}}$ the associated random walk. It is well known (see, e.g. [6, Lemma 4.1]) that, for any measurable $f: \mathbb{R}^{n+1} \rightarrow[0, \infty)$,

$$
\mathrm{E} f\left(S_{0}, \ldots, S_{n}\right)=\mathrm{E} \sum_{|u|=n} Y_{u} f(S(u \mid 0), S(u \mid 1), \ldots, S(u)),
$$

where, for $u=u_{1} \cdots u_{n}$, we write $u \mid k$ for individual $u_{1} \cdots u_{k}$, the ancestor of $u$ residing in the $k$ th generation. We note, in passing, that

$$
\varphi(t):=\mathrm{E}^{-t S_{1}}=m(1+t), \quad t \geq 0 .
$$

Define

$$
W_{n}:=\int_{\mathbb{R}} \mathrm{e}^{-x} \mathcal{M}_{n}(\mathrm{~d} x)=\sum_{|u|=n} Y_{u}, \quad n \in \mathbb{N}_{0}
$$


and denote the distribution of $W_{1}$ by $F$. Let $\mathcal{F}_{n}$ be the $\sigma$-field generated by the first $n$ generations, i.e. $\mathcal{F}_{n}=\sigma(\mathcal{M}(u):|u|<n)$, where $|u|<n$ means $u \in \mathbb{N}^{k}$ for some $k<n$.

It is well known and easy to check that $\left(W_{n}, \mathcal{F}_{n}\right)_{n \in \mathbb{N}_{0}}$ forms a nonnegative martingale and, thus, converges a.s. to a random variable $W$, say, with $\mathrm{E} W \leq 1$. This martingale, which is called the intrinsic martingale in the BRW, is of outstanding importance in the asymptotic analysis of the BRW (see, e.g. [9] and [15]). In this paper, we give sufficient conditions for the following statement to hold: for fixed $a>0$,

$$
\sum_{n \geq 0} \mathrm{e}^{a n}\left(W-W_{n}\right) \quad \text { converges a.s. }
$$

Clearly, (1.2) states that $\left(W_{n}\right)_{n \in \mathbb{N}_{0}}$ converges a.s. to $W$ exponentially fast.

There are already (at least) two articles which explore the rate of convergence of the intrinsic martingale in the BRW to its limit. In [2] necessary and sufficient conditions were found for the series in (1.2) to converge in $L_{p}, p>1$. Sufficient conditions for the almost-sure convergence of the series

$$
\sum_{n \geq 0} f(n)\left(W-W_{n}\right),
$$

where $f$ is a function regularly varying at $\infty$ with an index larger than -1 , were obtained in [12]. The results derived in both the paper at hand and [12] form a generalization of the results in [3, Section II.4], where the rate of the almost-sure convergence of the normalized supercritical Galton-Watson process to its limit was investigated. We want to remark that the scheme of our proofs borrows heavily from the ideas laid down in [3, Section II.4], but the technical details are much more involved. The source of complication can be easily understood: given $\mathcal{F}_{n}, W_{n+1}$ in the setting of Galton-Watson processes is just the sum of a finite number of i.i.d. random variables, whereas $W_{n+1}$ in the setting of the BRW is a weighted sum of a, possibly infinite, number of i.i.d. random variables.

Before stating our main results, we need some more notation and explanations. If $0<$ $\inf _{1 \leq \theta \leq 2} m^{1 / \theta}(\theta)<1$ then there exists a $\vartheta_{0} \in(1,2]$ such that $m^{1 / \vartheta_{0}}\left(\vartheta_{0}\right)=\inf _{1 \leq \theta \leq 2} m^{1 / \theta}(\theta)$. The derivative of the function $\theta \mapsto m^{1 / \theta}(\theta)$ is well defined and negative on $\left(1, \vartheta_{0}\right)$, and the left derivative is well defined and nonpositive on $\left(1, \vartheta_{0}\right]$. From this we conclude that the left derivative of $m$ (to be denoted by $m^{\prime}$ in what follows) is well defined and negative on $\left(1, \vartheta_{0}\right]$, i.e.

$$
m^{\prime}\left(\vartheta_{0}\right)<0
$$

Theorem 1.1. Let $a>0$ be given. Assume that

$$
\mathrm{e}^{a} m^{1 / r}(r) \leq 1 \quad \text { for some } r \in(1,2),
$$

and define $\vartheta$ to be the minimal $r>1$ such that $\mathrm{e}^{a r} m(r)=1$. Assume further that

$$
\mathrm{E} W_{1}^{\vartheta}<\infty
$$

and in the case where $a=-\log \inf _{r \geq 1} m^{1 / r}(r)$ (which implies that $\vartheta=\vartheta_{0}$ ) assume that

$$
-\frac{\log m\left(\vartheta_{0}\right)}{\vartheta_{0}}<-\frac{m^{\prime}\left(\vartheta_{0}\right)}{m\left(\vartheta_{0}\right)}
$$

Then $\sum_{n \geq 0} \mathrm{e}^{a n}\left(W-W_{n}\right)$ converges a.s. 


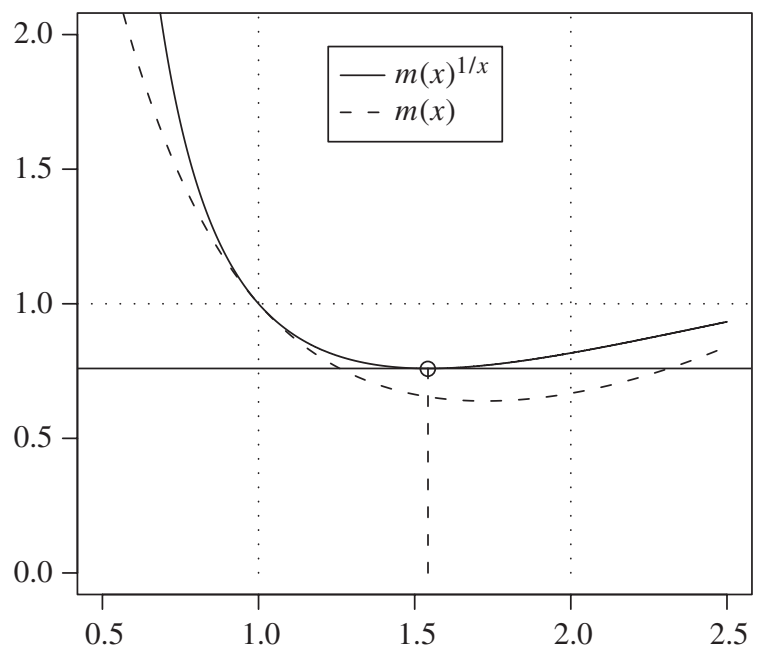

Figure 1: A typical situation in which Theorem 1.1 applies. Here $m(1)=1$, and $m$ is strictly decreasing in a right neighborhood of 1 . The bottom point of the graph of $m^{1 / x}(x)$ is marked by a circle. The vertical dashed line connects this point to the $x$-axis indicating the point $\vartheta_{0}$. The solid horizontal line and the dotted horizontal line at 1 indicate the interval of possible values of $\mathrm{e}^{-a}$ such that $a>0$ and $\mathrm{e}^{a} m^{1 / \vartheta_{0}} m\left(\vartheta_{0}\right)<1$. For those $a$ s, the assumptions of Theorem 1.1 are satisfied. The vertical dotted lines at 1 and 2 emphasize the importance of the interval $(1,2)$ in which $\vartheta_{0}$ is supposed to be located.

Remark 1.1. The point $\left(\vartheta_{0}, m^{1 / \vartheta_{0}}\left(\vartheta_{0}\right)\right)$ either belongs to the strictly decreasing branch of the graph $\left\{\left(x, m^{1 / x}(x)\right): x \in \mathfrak{D}(m)\right\}$, equivalently, $-\log m\left(\vartheta_{0}\right) / \vartheta_{0}<-m^{\prime}\left(\vartheta_{0}\right) / m\left(\vartheta_{0}\right)$, or it is the bottom point of that graph, which is equivalent to $\log m\left(\vartheta_{0}\right) / \vartheta_{0}=m^{\prime}\left(\vartheta_{0}\right) / m\left(\vartheta_{0}\right)$. From this we conclude that the theorem implies that (1.2) with $a=-\log m\left(\vartheta_{0}\right) / \vartheta_{0}$ holds when the former occurs. Intuitively, while the second situation is somewhat exceptional, the first situation illustrated in Figure 1 is more or less typical. In conclusion, the condition that $\mathrm{e}^{a} \inf _{1 \leq r \leq 2} m^{1 / r}(r)<1$ is 'typically' sufficient for (1.2) to hold. A similar remark with an obvious modification also applies to Theorem 2.1(a) and Theorem 2.2, below.

Remark 1.2. Let $p \in(1,2)$. By using a completely different argument, in [2] it was proved that the conditions

$$
\mathrm{E} W_{1}^{r}<\infty \text { and } \mathrm{e}^{a} m^{1 / r}(r)<1 \quad \text { for some } r \in[p, 2]
$$

are sufficient for the $\mathcal{L}_{p}$, and, hence, the almost-sure convergence of $\sum_{n \geq 0} \mathrm{e}^{a n}\left(W-W_{n}\right)$. Plainly, the conditions of our Theorem 1.1 are weaker.

Remark 1.3. Under the assumptions of Theorem 1.1, the martingale $\left(W_{n}\right)_{n \in \mathbb{N}_{0}}$ is uniformly integrable, equivalently, $\mathrm{P}(W>0)>0$. An ultimate criterion of uniform integrability of the intrinsic martingale was recently presented in [1], following earlier investigation in [5], [13], and [14].

Example 1.1. (Galton-Watson processes.) Suppose that $m:=\mathrm{E} J \in(1, \infty)$ and that $\mathrm{e}^{-X_{i}}=$ $m^{-1} \mathbf{1}_{\{i \leq J\}}, i \in \mathbb{N}$. Then $\left(W_{n}\right)_{n \in \mathbb{N}_{0}}$ forms a normalized supercritical Galton-Watson process. Choose $p$ and $q$ such that $p \in(1,2)$ and $1 / p+1 / q=1$. Theorem 4.1 of [3] proves that

$$
W-W_{n}=o\left(m^{-n / q}\right) \quad \text { a.s. as } n \rightarrow \infty
$$


if and only if

$$
\mathrm{E} W_{1}^{p}<\infty
$$

The sufficiency of condition (1.6) for (1.5) to hold follows from our Theorem 1.1. To see this, take $a=q^{-1} \log m$ and note that the equality $m(\theta)=m^{1-\theta}, \theta \geq 0$, implies that $\mathrm{e}^{a} m^{1 / r}(r)=$ $m^{1 / r-1 / p}<1$ for $r>p$. Therefore, (1.3) holds (with strict inequality). Furthermore, $\vartheta$ defined in Theorem 1.1 equals $p \in(1,2)$ in the present situation, which shows that $(1.4)$ holds. By Theorem 1.1, (1.5) holds.

The rest of the paper is organized as follows. In Section 2 we present auxiliary renewaltheoretic results which correct, generalize, and simplify some earlier results from [10] and [11]. Statements of this section are an important ingredient of the proof of Theorem 1.1, which is given in Section 3.

\section{Ultimate results for the exponential renewal function}

For a random variable $T$ with proper distribution which we assume to be nondegenerate at 0 , let $\psi$ be its Laplace transform:

$$
\psi:[0, \infty) \rightarrow(0, \infty], \quad \psi(t):=\mathrm{Ee}^{-t T} .
$$

In what follows, we denote by $\psi^{\prime}$ the left derivative of $\psi$.

Set $R:=-\log \inf _{t \geq 0} \psi(t)$. Then $R \geq 0$ since $\psi(0)=1$, and unless $T \geq 0$ a.s., the infimum in the definition of $R$ is attained, i.e. there exists some $\gamma_{0} \in[0, \infty)$ such that $\psi\left(\gamma_{0}\right)=\mathrm{e}^{-R}$. Note that $\gamma_{0}=0$ is equivalent to $R=0$ since we assume that the distribution of $T$ is nondegenerate at 0 . When $R>0$ and $a \in(0, R]$, let $\gamma$ denote the minimal (finite) $t>0$ satisfying $\psi(t)=\mathrm{e}^{-a}$ if such a $t$ exists. Note that $\gamma=\gamma_{0}$ if $a=R$ and the infimum is attained. Let $\left(T_{n}\right)_{n \in \mathbb{N}_{0}}$ be a zero-delayed random walk with a step distributed like $T$. Whenever $\gamma$ as above exists, we use it to define a new probability measure $\mathrm{P}_{\gamma}$ such that

$$
\mathrm{P}_{\gamma}\left(T_{n} \in A\right)=\psi(\gamma)^{-n} \mathrm{Ee}^{-\gamma T_{n}} \mathbf{1}_{\left\{T_{n} \in A\right\}}, \quad n \in \mathbb{N}_{0},
$$

for any Borel set $A \subseteq \mathbb{R}$. As a consequence of $\mathrm{E}_{\gamma} \mathrm{e}^{\gamma T_{1}}=\psi(\gamma)^{-1}<\infty$, we have

$$
\mathrm{E}_{\gamma} T_{1}^{+}<\infty \text {. }
$$

Since $\psi$ is nonincreasing on $\left[0, \gamma_{0}\right]$, we conclude that if $\gamma_{0}>0$ then

$$
\mathrm{E}_{\gamma_{0}} T_{1}=\mathrm{Ee}^{-\gamma_{0} T} T=-\psi^{\prime}\left(\gamma_{0}\right)
$$

should be nonnegative and finite in view of (2.2).

In the first theorem in this section we investigate the finiteness of the exponential renewal function

$$
V(x):=\sum_{n \geq 0} \mathrm{e}^{a n} \mathrm{P}\left(T_{n} \leq x\right), \quad x \in \mathbb{R} .
$$

Theorem 2.1. Assume that $\mathrm{P}(T=0) \neq 1$, and let $a>0$ be given.

(a) Assume that $\mathrm{P}(T<0)>0$.

(i) If $a \in(0, R)$ then $V(x)$ is finite for every $x \in \mathbb{R}$. 
(ii) If $a=R>0$ and

$$
-\psi^{\prime}\left(\gamma_{0}\right)=\mathrm{Ee}^{-\gamma_{0} T} T>0
$$

then $V(x)$ is finite for every $x \in \mathbb{R}$.

(iii) If $a>R$ then $V(x)=+\infty$ for all $x \in \mathbb{R}$.

(iv) If $a=R>0$ and $\psi^{\prime}\left(\gamma_{0}\right)=0$ (equivalently, if (2.3) does not hold), then $V(x)=$ $+\infty$ for all $x \in \mathbb{R}$.

(b) Assume that $\mathrm{P}(T>0)=1$. Then $V(x)$ is finite for every $x \in \mathbb{R}$.

(c) Assume that $\mathrm{P}(T \geq 0)=1$ and $\beta:=\mathrm{P}(T=0)>0$. Then $R=-\log \beta$ and if $a \in(0, R)$ then $V(x)$ is finite for every $x \in \mathbb{R}$, and if $a \geq R$ then $V(x)$ is infinite for all $x \geq 0$.

Theorem 2.1 constitutes a generalization of Theorem B of [10], but can also be partly deduced (excluding the case $a=R$ ) from the more general Theorem 2 of [8]. Our contribution here is a streamlined derivation of the exact value of $R$, a simple proof of dichotomy $a<R$ versus $a>R$, and an investigation of the most delicate case $a=R$.

The main tool for the analysis in Section 3 is the following result, which provides the asymptotic behavior of the exponential renewal function $V(x)$. Note in advance that Theorem 2.2 will be applied to $\left(S_{n}-a n\right)_{n \in \mathbb{N}_{0}}$, where $\left(S_{n}\right)_{n \in \mathbb{N}_{0}}$ is the associated random walk of the given BRW.

Theorem 2.2. Let $a>0$ be given. Assume that either $a \in(0, R)$ or $a=R$ and (2.3) holds. Then, with $\gamma$ being the minimal $t>0$ satisfying $\psi(t)=\mathrm{e}^{-a}$,

$$
V(x) \sim \frac{\mathrm{e}^{-a}}{\gamma\left(-\psi^{\prime}(\gamma)\right)} \mathrm{e}^{\gamma x} \text { as } x \rightarrow \infty
$$

if $\left(T_{n}\right)_{n \in \mathbb{N}_{0}}$ is a nonarithmetic random walk, and

$$
V(\lambda n) \sim \frac{\lambda \mathrm{e}^{-a}}{\left(1-\mathrm{e}^{-\lambda \gamma}\right)\left(-\psi^{\prime}(\gamma)\right)} \mathrm{e}^{\gamma \lambda n} \text { as } n \rightarrow \infty
$$

if $\left(T_{n}\right)_{n \in \mathbb{N}_{0}}$ is arithmetic with span $\lambda>0$. Moreover, in the arithmetic case,

$$
V(\lambda n)-V(\lambda(n-1)) \sim \frac{\lambda \mathrm{e}^{-a}}{\left(-\psi^{\prime}(\gamma)\right)} \mathrm{e}^{\gamma \lambda n} \text { as } n \rightarrow \infty .
$$

Remark 2.1. (a) Theorem 2.2 describes the asymptotics of $V(x)$ whenever it is finite.

(b) Provided that $a<R$ or $a=R$ and (2.3) holds, the equation $\psi(t)=\mathrm{e}^{-a}$ has positive solutions.

Theorem 2.2 is a generalization and correction of Theorem 4 of [11], the differences being that

- we do not assume that $\mathrm{E}|T|$ is finite;

- the exponential (wrong) rate $a / \mathrm{E} T$ claimed in [11] under the assumption that $\mathrm{E} T \in$ $(0, \infty)$ is replaced by the rate $\gamma$ in the nonarithmetic case, and a similar substitution is proved to hold true in the arithmetic case;

- unlike Heyde [11] we treat, among others, the boundary case $a=R$. 
We think that an error in the proof of Theorem 4 of [11] appears at the end of page 706 where the dependence of the real number $\xi(n) \in(\beta, \mu)$ on $n$ cannot be ignored since possibly $\xi(n) \rightarrow \mu$ and then $\mathrm{P}\left(S_{n} \leq \xi(n)\right)$ does not necessarily decay at an exponential rate (retaining the original notation from [11]).

Proof of Theorem 2.1. (a) (i) If $R=0$ then condition $a \in(0, R)$ cannot hold. So assume that $R>0, a \in(0, R)$, and pick any $x \in \mathbb{R}$. With $\gamma_{0}$ defined at the beginning of the section, choose $r \in\left(0, \gamma_{0}\right)$ such that $a<-\log \psi(r)$. Now use Markov's inequality to obtain

$$
\sum_{n \geq 0} \mathrm{e}^{a n} \mathrm{P}\left(T_{n} \leq x\right) \leq \sum_{n \geq 0} \mathrm{e}^{a n} \mathrm{e}^{r x} \mathrm{Ee}^{-r T_{n}}=\mathrm{e}^{r x} \sum_{n \geq 0} \mathrm{e}^{n(a+\log \psi(r))}<\infty,
$$

which proves the assertion under assumption (i).

Consider parts (ii) and (iv) of (a). Assume that $a=R>0$. The function $g(y):=$ $\mathrm{e}^{-\gamma_{0} y} \mathbf{1}_{[0, \infty)}(y)$ is directly Riemann integrable. If (2.3) holds then the random walk $\left(T_{n}\right)_{n \in \mathbb{N}_{0}}$ is transient under $\mathrm{P}_{\gamma_{0}}$, the probability measure defined in (2.1), and if (2.3) does not hold then $\left(T_{n}\right)_{n \in \mathbb{N}_{0}}$ is recurrent under $\mathrm{P}_{\gamma_{0}}$. As a consequence, the renewal measure $U_{\gamma_{0}}$ of $\left(T_{n}\right)_{n \in \mathbb{N}_{0}}$ under $\mathrm{P}_{\gamma_{0}}$ satisfies $U_{\gamma_{0}}(I)<\infty$ if (2.3) holds and $U_{\gamma_{0}}(I)=\infty$ if (2.3) does not hold for any bounded open nonempty interval $I$ if $\left(T_{n}\right)$ is nonarithmetic, and for any bounded open nonempty interval $I$ which contains some point $n \lambda, n \in \mathbb{Z}$, if $\left(T_{n}\right)$ is arithmetic with span $\lambda$. Therefore, if (2.3) holds then

$$
\begin{aligned}
\sum_{n \geq 0} \mathrm{e}^{a n} \mathrm{P}\left(T_{n} \leq x\right) & =\sum_{n \geq 0} \mathrm{E}_{\gamma_{0}} \mathrm{e}^{\gamma_{0} T_{n}} \mathbf{1}_{\left\{T_{n} \leq x\right\}} \\
& =\mathrm{e}^{\gamma_{0} x} \sum_{n \geq 0} \mathrm{E}_{\gamma_{0}} g\left(x-T_{n}\right) \\
& <\infty, \quad x \in \mathbb{R} .
\end{aligned}
$$

Whereas, if (2.3) does not hold then

$$
\sum_{n \geq 0} \mathrm{e}^{a n} \mathrm{P}\left(T_{n} \leq x\right)=\mathrm{e}^{\gamma_{0} x} \sum_{n \geq 0} \mathrm{E}_{\gamma_{0}} g\left(x-T_{n}\right)=\infty, \quad x \in \mathbb{R} .
$$

(Note that this argument with $\gamma_{0}$ replaced by $\gamma$ also applies in the situation of (a)(i).)

(iii) To complete the proof of (a), it remains to check that $V(x)=+\infty$ for all $x \in \mathbb{R}$ provided that $a>R$. Note that the case $R=0$ is not excluded and is equivalent to $\gamma_{0}=0$. Furthermore, note that $\psi$ assumes its infimum on $[0, \infty)$ since we assume that $\mathrm{P}(T<0)>0$. Recall that the unique minimizer of $\psi$ is denoted by $\gamma_{0}$ and that $\psi^{\prime}\left(\gamma_{0}\right)$, the left derivative of $\psi$ at 0 , exists and is less than or equal to 0 if $\gamma_{0}>0$.

Subcase (iii.1): $\gamma_{0}>0$. If $\psi^{\prime}\left(\gamma_{0}\right)<0$ then, for any $c>0$, we consider a zero-delayed random walk, $\left(T_{c, n}\right)_{n \in \mathbb{N}_{0}}$ say, with steps distributed like $T \mathbf{1}_{\{T \geq-c\}}$. Set $\psi_{c}(t):=\mathrm{Ee}^{-t T_{c, 1}}$, and note that $\psi_{c}$ is finite on $[0, \infty)$ and that $R_{c}:=-\log \inf _{t \geq 0} \psi_{c}(t) \geq R$. If $c$ is large enough, $\psi_{c}(t) \rightarrow \infty$ as $t \rightarrow \infty$. Thus, $\psi_{c}$ has a unique minimizer on $[0, \infty), \gamma_{c}$ say, and $\psi_{c}^{\prime}\left(\gamma_{c}\right)=0$. It is easily seen that $\gamma_{0} \leq \gamma_{c}$ and that $\gamma_{c} \downarrow \gamma_{0}$ as $c \uparrow \infty$. Some elementary analysis now shows that $R_{c}$ converges to $R$ as $c \rightarrow \infty$. Moreover,

$$
\sum_{n \geq 0} \mathrm{e}^{a n} \mathrm{P}\left(T_{n} \leq x\right) \geq \sum_{n \geq 0} \mathrm{e}^{a n} \mathrm{P}\left(T_{c, n} \leq x\right), \quad x \in \mathbb{R} .
$$

Therefore, if we can prove that, provided $a>R_{c}$, the series $\sum_{n \geq 0} \mathrm{e}^{a n} \mathrm{P}\left(T_{c, n} \leq x\right)$ diverges, then this will imply (after choosing $c$ sufficiently large) that, provided $a>R$, the series 
$\sum_{n \geq 0} \mathrm{e}^{a n} \mathrm{P}\left(T_{n} \leq x\right)$ diverges. Thus, we have shown that, without loss of generality, we can work under the additional assumption that $\psi^{\prime}\left(\gamma_{0}\right)=0$. The condition $a>R$ now reads as $\psi\left(\gamma_{0}\right)>\mathrm{e}^{-a}$. By using the probability measure $\mathrm{P}_{\gamma_{0}}$ defined in (2.1) we conclude that $\mathrm{E}_{\gamma_{0}} T_{1}=0$. Hence, the random walk $\left(T_{n}\right)_{n \in \mathbb{N}_{0}}$ is recurrent under $\mathrm{P}_{\gamma_{0}}$. As a consequence, the renewal measure $U_{\gamma_{0}}$ of $\left(T_{n}\right)_{n \in \mathbb{N}_{0}}$ under $\mathrm{P}_{\gamma_{0}}$ satisfies $U_{\gamma_{0}}(I)=\infty$ for any open nonempty interval $I$ if $\left(T_{n}\right)$ is nonarithmetic, and for any open nonempty interval $I$ which contains some point $n \lambda, n \in \mathbb{Z}$, if $\left(T_{n}\right)$ is arithmetic with $\operatorname{span} \lambda$, respectively. As a consequence,

$$
\begin{aligned}
\sum_{n \geq 0} \mathrm{e}^{a n} \mathrm{P}\left(T_{n} \leq x\right) & =\sum_{n \geq 0}\left(\psi\left(\gamma_{0}\right) \mathrm{e}^{a}\right)^{n} \mathrm{E}_{\gamma_{0}} \mathrm{e}^{\gamma_{0} T_{n}} \mathbf{1}_{\left\{T_{n} \leq x\right\}} \\
& \geq \int_{(-\infty, x]} \mathrm{e}^{\gamma_{0} y} U_{\gamma_{0}}(\mathrm{~d} y) \\
& =\infty
\end{aligned}
$$

for every $x \in \mathbb{R}$.

Subcase (iii.2): $\gamma_{0}=0$. We have $\psi(t) \in(1, \infty]$ for all $t>0$. If $T_{n} \rightarrow-\infty$ a.s. then $\mathrm{P}\left(T_{n} \leq x\right) \rightarrow 1$ as $n \rightarrow \infty$, and the infinite series $\sum_{n>0} \mathrm{e}^{a n} \mathrm{P}\left(T_{n} \leq x\right)$ diverges. Thus, we are left with the situation that either $T_{n} \rightarrow \infty$ a.s. or $\left(T_{n}\right)_{n \in \mathbb{N}_{0}}$ oscillates. In both cases, $\mathrm{E} T_{1, c} \in(0, \infty]$, where $T_{1, c}$ is defined as above, and the Laplace transform $\psi_{c}$ of $T_{c, 1}$ is finite on $[0, \infty)$ and assumes its minimum at some $\gamma_{c}>0$ satisfying $\psi_{c}^{\prime}\left(\gamma_{c}\right)=0$. Now we can argue as in subcase (iii.1) to show that $\sum_{n \geq 0} \mathrm{e}^{a n} \mathrm{P}\left(T_{n} \leq x\right)=\infty$ for all $x \in \mathbb{R}$.

(b) In this case $R=+\infty$. Choose any $x>0(V(x)=0$ for $x \leq 0)$, choose $r>0$ such that $a<-\log \psi(r)$, and proceed in the same way as under (a)(i).

(c) Note that we have $\beta \in(0,1)$. Choose $a \in(0,-\log \beta)$. The subsequent proof literally repeats that given under assumption (a)(i).

Conversely, $\mathrm{P}\left(T_{n}=0\right)=\beta^{n}, n \in \mathbb{N}_{0}$. Therefore, if $a \geq-\log \beta$ then $V(0)=+\infty$, which implies that $V(x)=+\infty$ for all $x \geq 0$.

Proof of Theorem 2.2. By Theorem 2.1, $V(x)<\infty$ for every $x \in \mathbb{R}$. Under $\mathrm{P}_{\gamma}$, the probability measure defined in $(2.1),\left(T_{n}\right)_{n \in \mathbb{N}_{0}}$ forms a random walk with Laplace transform

$$
\psi_{\gamma}(t)=\mathrm{E}_{\gamma} \mathrm{e}^{-t T_{1}}=\mathrm{e}^{a} \mathrm{Ee}^{-(\gamma+t) T}=\mathrm{e}^{a} \psi(\gamma+t)
$$

and drift

$$
v_{\gamma}=-\psi_{\gamma}^{\prime}(0)=-\mathrm{e}^{a} \psi^{\prime}(\gamma) \in(0, \infty)
$$

For $x \in \mathbb{R}$, we write $V(x)$ in the following form:

$$
V(x)=\sum_{n \geq 0} \mathrm{E}_{\gamma} \mathrm{e}^{\gamma T_{n}} \mathbf{1}_{\left\{T_{n} \leq x\right\}}=\int_{(-\infty, x]} \mathrm{e}^{\gamma y} U_{\gamma}(\mathrm{d} y)=: \mathrm{e}^{\gamma x} Z(x),
$$

where $U_{\gamma}$ denotes the renewal measure of the process $\left(T_{n}\right)_{n \geq 0}$ under $\mathrm{P}_{\gamma}$. Assume that $\left(T_{n}\right)_{n \in \mathbb{N}_{0}}$ is nonarithmetic. Since

$$
Z(x)=\mathrm{e}^{-\gamma x} \int_{(-\infty, x]} \mathrm{e}^{\gamma y} U_{\gamma}(\mathrm{d} y)=\int \mathrm{e}^{-\gamma(x-y)} \mathbf{1}_{[0, \infty)}(x-y) U_{\gamma}(\mathrm{d} y)
$$

and the function $x \mapsto \mathrm{e}^{-\gamma x} \mathbf{1}_{[0, \infty)}(x)$ is directly Riemann integrable, we can invoke the key renewal theorem on the whole line to conclude that

$$
\mathrm{e}^{-\gamma x} \sum_{n \geq 0} \mathrm{e}^{a n} \mathrm{P}\left(T_{n} \leq x\right)=Z(x) \stackrel{x \rightarrow \infty}{\longrightarrow} \frac{1}{v_{\gamma}} \int_{0}^{\infty} \mathrm{e}^{-\gamma y} \mathrm{~d} y=\frac{1}{\gamma v_{\gamma}},
$$


where we have used the fact that $v_{\gamma}>0$. This, in combination with (2.7), immediately implies (2.4).

Asymptotics (2.5) in the arithmetic case can be treated similarly. Finally, (2.6) follows by an application of (2.5) to

$$
\mathrm{e}^{-\gamma \lambda n}(V(\lambda n)-V(\lambda(n-1)))=\mathrm{e}^{-\gamma \lambda n} V(\lambda n)-\mathrm{e}^{-\gamma \lambda}\left(\mathrm{e}^{-\gamma \lambda(n-1)} V(\lambda(n-1))\right) .
$$

\section{Proof of Theorem 1.1}

For any $u \in \mathbb{V}$, let $W_{1}(u)$ denote a copy of $W_{1}$ but based on the point process $\mathcal{M}(u)$ instead of $\mathcal{M}=\mathcal{M}(\varnothing)$, that is, if $W_{1}=\psi(\mathcal{M})$ for an appropriate measurable function $\psi$ then $W_{1}(u):=\psi(\mathcal{M}(u))$. In this situation, let

$$
\begin{aligned}
\tilde{W}_{n+1} & :=\sum_{|u|=n} Y_{u} W_{1}(u) \mathbf{1}_{\left\{\mathrm{e}^{a n} Y_{u} W_{1}(u) \leq 1\right\}} \\
\text { and } \quad R_{n} & :=\mathrm{E}\left(W_{n}-\widetilde{W}_{n+1} \mid \mathcal{F}_{n}\right), \quad n \in \mathbb{N}_{0} .
\end{aligned}
$$

For $a>0$, define a measure $V_{a}$ on the Borel subsets of $\mathbb{R}^{+}$by

$$
V_{a}(x):=V_{a}((0, x]):=\sum_{n \geq 0} \mathrm{e}^{a n} \mathrm{P}\left(S_{n}-a n \leq \log x\right), \quad x>0,
$$

where $\left(S_{n}\right)_{n \in \mathbb{N}_{0}}$ is the associated random walk of the given BRW.

We now present a result on the asymptotic behavior of $V_{a}$ and two integrals involving $V_{a}$ which play an important role in the proof of Theorem 1.1.

Lemma 3.1. Let $a>0$ be given. Assume that $\mathrm{e}^{a} m^{1 / r}(r) \leq 1$ for some $r>1$, and define $\vartheta$ to be the minimal $r>1$ such that $\mathrm{e}^{a} m^{1 / r}(r)=1$. In the case where $a=-\log \inf _{r \geq 1} m^{1 / r}(r)$ (which implies that $\vartheta=\vartheta_{0}$ ) assume further that

$$
-\frac{\log m\left(\vartheta_{0}\right)}{\vartheta_{0}}<-\frac{m^{\prime}\left(\vartheta_{0}\right)}{m\left(\vartheta_{0}\right)}
$$

Then, as $x \rightarrow \infty$,

$$
\begin{gathered}
V_{a}(x) \sim \frac{x^{\vartheta-1}}{(\vartheta-1)\left(\mathrm{e}^{a \vartheta}\left(-m^{\prime}(\vartheta)\right)-a\right)}, \\
\int_{(0, x]} y V_{a}(\mathrm{~d} y) \sim \frac{x^{\vartheta}}{\vartheta\left(\mathrm{e}^{a \vartheta}\left(-m^{\prime}(\vartheta)\right)-a\right)},
\end{gathered}
$$

if the random walk $\left(S_{n}\right)_{n \in \mathbb{N}_{0}}$ is nonarithmetic. If $\left(S_{n}-\text { an }\right)_{n \in \mathbb{N}_{0}}$ has span $\lambda_{a}>0$ then, analogously, as $n \rightarrow \infty$,

$$
\begin{gathered}
V_{a}\left(\mathrm{e}^{\lambda_{a} n}\right) \sim \frac{\lambda_{a} \mathrm{e}^{(\vartheta-1) \lambda_{a} n}}{\left(1-\mathrm{e}^{-\lambda_{a}(\vartheta-1)}\right)\left(\mathrm{e}^{a \vartheta}\left(-m^{\prime}(\vartheta)\right)-a\right)}, \\
\int_{\left(0, \mathrm{e}^{\lambda_{a} n}\right]} y V_{a}(\mathrm{~d} y) \sim \frac{\lambda_{a} \mathrm{e}^{\vartheta \lambda_{a} n}}{\left(1-\mathrm{e}^{-\lambda_{a} \vartheta}\right)\left(\mathrm{e}^{a \vartheta}\left(-m^{\prime}(\vartheta)\right)-a\right)} .
\end{gathered}
$$

Furthermore, if $\vartheta<2$ then in the nonarithmetic case, as $x \rightarrow \infty$,

$$
\int_{(x, \infty)} y^{-1} V_{a}(\mathrm{~d} y) \sim \frac{x^{\vartheta-2}}{(2-\vartheta)\left(\mathrm{e}^{a \vartheta}\left(-m^{\prime}(\vartheta)\right)-a\right)},
$$


whereas in the arithmetic case, as $n \rightarrow \infty$,

$$
\int_{\left[\mathrm{e}^{\lambda_{a} n}, \infty\right)} y^{-1} V_{a}(\mathrm{~d} y) \sim \frac{\lambda_{a} \mathrm{e}^{(\vartheta-2) \lambda_{a} n}}{\left(1-\mathrm{e}^{(\vartheta-2) \lambda_{a}}\right)\left(a^{a \vartheta}\left(-m^{\prime}(\vartheta)\right)-a\right)} .
$$

Proof. Let $\varphi$ and $\varphi_{a}$ be the Laplace transforms of the increment distributions of the associated random walk $\left(S_{n}\right)_{n \in \mathbb{N}_{0}}$ defined by (1.1) and of the shifted random walk $\left(S_{n}-a n\right)_{n \in \mathbb{N}_{0}}$, respectively. Our aim is to check that, under the assumptions of the lemma, Theorem 2.2 applies to the random walk $\left(S_{n}-a n\right)_{n \in \mathbb{N}_{0}}$ with $\psi=\varphi_{a}$ and $R=-\log \inf _{t \geq 0} \varphi_{a}(t)$.

By definition, $\varphi_{a}(t)=\mathrm{e}^{a t} \varphi(t)=\mathrm{e}^{a t} m(1+t)$, which implies that

$$
\mathrm{e}^{a} \varphi_{a}(r-1)=\mathrm{e}^{a r} m(r) \leq 1 .
$$

Therefore, in the notation of Theorem 2.2, the condition that $a \leq R$ holds. In the case $a=$ $-\log \inf _{t \geq 1} m^{1 / t}(t)$, we have $a=-\log \inf _{t \geq 0} \varphi_{a}(t)=R$ in view of (3.8). With $\psi=\varphi_{a}$, $\gamma_{0}$ defined at the beginning of Section 2 equals $\vartheta_{0}-1$. Therefore, condition (2.3) reads $\varphi_{a}^{\prime}\left(\vartheta_{0}-1\right)<0$ and is a consequence of (3.1). In any case, Theorem 2.2 applies with $\gamma$ being the minimal $t>0$ satisfying $\varphi_{a}(t)=\mathrm{e}^{-a}$, that is, $\gamma=\vartheta-1$, and yields

$$
V_{a}(x) \sim \frac{\mathrm{e}^{-a}}{(\vartheta-1)\left(-\varphi_{a}^{\prime}(\vartheta-1)\right)} x^{\vartheta-1}, \quad x \rightarrow \infty,
$$

in the case where $\left(S_{n}-a n\right)_{n \in \mathbb{N}_{0}}$ is nonarithmetic, and

$$
V_{a}\left(\mathrm{e}^{\lambda_{a} n}\right) \sim \frac{\lambda_{a} \mathrm{e}^{-a}}{\left(1-\mathrm{e}^{-\lambda_{a}(\vartheta-1)}\right)\left(-\varphi_{a}^{\prime}(\vartheta-1)\right)} \mathrm{e}^{(\vartheta-1) \lambda_{a} n}, \quad n \rightarrow \infty,
$$

in the case where $\left(S_{n}-a n\right)_{n \in \mathbb{N}_{0}}$ is arithmetic with span $\lambda_{a}$.

Now first note that (3.9) proves (3.2) and that (3.10) implies (3.4). Secondly, in the nonarithmetic case, asymptotics (3.3) and (3.6) follow from (3.2) by integration by parts and subsequent application of Propositions 1.5 .8 and 1.5.10 of [7], respectively. Finally, in the lattice case, asymptotics (3.5) and (3.7) follow by an elementary analysis from (3.4) and the corresponding asymptotic for $V_{a}\left(\mathrm{e}^{\lambda_{a} n}\right)-V_{a}\left(\mathrm{e}^{\lambda_{a}(n-1)}\right)$, which can be derived from (2.6). We omit the details.

Lemma 3.2. Let $a>0$ be given. Assume that $\mathrm{e}^{a} m^{1 / r}(r) \leq 1$ for some $r>1$, and define $\vartheta$ to be the minimal $r>1$ such that $\mathrm{e}^{a} m^{1 / r}(r)=1$. In the case where $a=-\log \inf _{r \geq 1} m^{1 / r}(r)$ (which implies that $\vartheta=\vartheta_{0}$ ) assume further that

$$
-\frac{\log m\left(\vartheta_{0}\right)}{\vartheta_{0}}<-\frac{m^{\prime}\left(\vartheta_{0}\right)}{m\left(\vartheta_{0}\right)}
$$

Then $\mathrm{E} W_{1}^{\vartheta}<\infty$ implies that

$$
\sum_{n \geq 0} \mathrm{P}\left(\tilde{W}_{n+1} \neq W_{n+1}\right)<\infty \text { and } \mathrm{E} \sum_{n \geq 0} \mathrm{e}^{a n} R_{n}<\infty .
$$

If, moreover, $\vartheta<2$ then $\left(M_{n}\right)_{n \in \mathbb{N}_{0}}$ is an $\mathcal{L}^{2}$-bounded martingale, where

$$
M_{n}:=\sum_{k=0}^{n} \mathrm{e}^{a k}\left(\tilde{W}_{k+1}-W_{k}+R_{k}\right), \quad n \in \mathbb{N}_{0} .
$$


Remark 3.1. Note that the second infinite series in (3.11) is well defined since all summands are nonnegative. Indeed, for any $n \in \mathbb{N}_{0}$, by the independence of $W_{1}(v)$ and $\mathcal{F}_{n}$ for $|v|=n$,

$$
\begin{aligned}
R_{n} & =\sum_{|v|=n} Y_{v} \mathrm{E}\left(1-W_{1}(v) \mathbf{1}_{\left\{\mathrm{e}^{a n} Y_{v} W_{1}(v) \leq 1\right\}} \mid \mathcal{F}_{n}\right) \\
& =\sum_{|v|=n} Y_{v} \mathrm{E}\left(W_{1}(v)-W_{1}(v) \mathbf{1}_{\left\{\mathrm{e}^{a n} Y_{v} W_{1}(v) \leq 1\right\}} \mid \mathcal{F}_{n}\right) \\
& =\sum_{|v|=n} Y_{v} \int_{\left(e^{-a n} Y_{v}^{-1}, \infty\right)} x F(\mathrm{~d} x) \\
& \geq 0 \quad \text { a.s. }
\end{aligned}
$$

where $F$ denotes the distribution of $W_{1}$.

Proof of Lemma 3.2. By using (1.1) we have

$$
\begin{aligned}
\sum_{n \geq 0} \mathrm{P}\left(\widetilde{W}_{n+1} \neq W_{n+1}\right) & \leq \sum_{n \geq 0} \mathrm{E} \sum_{|v|=n} \mathrm{P}\left(\mathrm{e}^{a n} Y_{v} W_{1}(v)>1 \mid \mathcal{F}_{n}\right) \\
& =\sum_{n \geq 0} \mathrm{E} \sum_{|v|=n} Y_{v} \mathrm{e}^{S(v)} \int \mathbf{1}_{\left(\mathrm{e}^{-a n} \mathrm{e}^{S(v)}, \infty\right)}(x) F(\mathrm{~d} x) \\
& =\sum_{n \geq 0} \mathrm{Ee}^{S_{n}} \int \mathbf{1}_{\left(\mathrm{e}^{S_{n}-a n}, \infty\right)}(x) F(\mathrm{~d} x) \\
& =\int \sum_{n \geq 0} \mathrm{Ee}^{S_{n}} \mathbf{1}_{\left\{\mathrm{e}^{S_{n}-a n}<x\right\}} F(\mathrm{~d} x) \\
& =\int \sum_{n \geq 0} \mathrm{e}^{a n} \mathrm{Ee}^{S_{n}-a n} \mathbf{1}_{(0, x)}\left(\mathrm{e}^{S_{n}-a n}\right) F(\mathrm{~d} x) \\
& =\iint_{(0, x)} y V_{a}(\mathrm{~d} y) F(\mathrm{~d} x) .
\end{aligned}
$$

Using (3.3) or (3.5), respectively, yields

$$
\sum_{n \geq 0} \mathrm{P}\left(\tilde{W}_{n+1} \neq W_{n+1}\right) \leq \int O\left(x^{\vartheta}\right) F(\mathrm{~d} x)<\infty .
$$

Concerning the second series in (3.11), we obtain, using the calculations from Remark 3.1,

$$
\begin{aligned}
\mathrm{E} \sum_{n \geq 0} \mathrm{e}^{a n} R_{n} & =\sum_{n \geq 0} \mathrm{e}^{a n} \mathrm{E} \sum_{|v|=n} Y_{v} \int_{\left(\mathrm{e}^{-a n} Y_{v}^{-1}, \infty\right)} x F(\mathrm{~d} x) \\
& =\sum_{n \geq 0} \mathrm{e}^{a n} \mathrm{E} \int_{\left(\mathrm{e}^{S_{n}-a n}, \infty\right)} x F(\mathrm{~d} x) \\
& =\int x \sum_{n \geq 0} \mathrm{e}^{a n} \mathrm{P}\left(S_{n}-a n<\log x\right) F(\mathrm{~d} x) \\
& \leq \int x V_{a}(x) F(\mathrm{~d} x) .
\end{aligned}
$$


In view of (3.2) and (3.4), we conclude that

$$
\mathrm{E} \sum_{n \geq 0} \mathrm{e}^{a n} R_{n} \leq \int x O\left(x^{\vartheta-1}\right) F(\mathrm{~d} x)=\int O\left(x^{\vartheta}\right) F(\mathrm{~d} x)<\infty .
$$

Now we turn to the final assertion of the lemma. Since $R_{n}=\mathrm{E}\left(W_{n}-\widetilde{W}_{n+1} \mid \mathcal{F}_{n}\right)$, we have $\widetilde{W}_{n+1}-W_{n}+R_{n}=\widetilde{W}_{n+1}-\mathrm{E}\left(\widetilde{W}_{n+1} \mid \mathcal{F}_{n}\right)$ a.s. In particular, $\left(M_{n}\right)_{n \geq 0}$ constitutes a martingale. It remains to prove that $\left(M_{n}\right)_{n \in \mathbb{N}_{0}}$ is $\mathcal{L}^{2}$-bounded. For this purpose, note that

$$
\begin{aligned}
\mathrm{E}\left(\mathrm{e}^{a n}\left(\widetilde{W}_{n+1}-W_{n}+R_{n}\right)\right)^{2} & =\mathrm{e}^{2 a n} \mathrm{E}\left(\widetilde{W}_{n+1}-W_{n}+R_{n}\right)^{2} \\
& =\mathrm{e}^{2 a n} \mathrm{E} \operatorname{var}\left(\widetilde{W}_{n+1} \mid \mathcal{F}_{n}\right) \\
& \leq \mathrm{e}^{2 a n} \mathrm{E} \sum_{|v|=n} Y_{v}^{2} \int_{\left(0, \mathrm{e}^{-a n} Y_{v}^{-1}\right]} x^{2} F(\mathrm{~d} x) .
\end{aligned}
$$

Thus,

$$
\begin{aligned}
\sum_{n \geq 0} \mathrm{E} & \left(\mathrm{e}^{a n}\left(\tilde{W}_{n+1}-W_{n}+R_{n}\right)\right)^{2} \\
& \leq \sum_{n \geq 0} \mathrm{e}^{2 a n} \mathrm{E} \sum_{|v|=n} Y_{v}^{2} \int_{\left(0, \mathrm{e}^{-a n} Y_{v}^{-1}\right]} x^{2} F(\mathrm{~d} x) \\
& =\sum_{n \geq 0} \mathrm{e}^{a n} \mathrm{E} \sum_{|v|=n} Y_{v} \mathrm{e}^{a n-S(v)} \int_{\left(0, \mathrm{e}^{S(v)-a n}\right]} x^{2} F(\mathrm{~d} x) \\
& =\sum_{n \geq 0} \mathrm{e}^{a n} \mathrm{Ee}^{-\left(S_{n}-a n\right)} \int_{\left(0, \mathrm{e}^{\left.S_{n}-a n\right]}\right]} x^{2} F(\mathrm{~d} x) \\
& =\int_{(0, \infty)} x^{2} \sum_{n \geq 0} \mathrm{e}^{a n} \mathrm{E}^{-\left(S_{n}-a n\right)} \mathbf{1}_{\left\{\mathrm{e}^{S_{n}-a n} \geq x\right\}} F(\mathrm{~d} x) \\
& =\int_{(0, \infty)} x^{2} \int_{[x, \infty)} y^{-1} V_{a}(\mathrm{~d} y) F(\mathrm{~d} x) .
\end{aligned}
$$

Now use (3.6) or (3.7) to obtain the finiteness of the last expression.

The proof of the next result can be found in Remark 3.2 of [2].

Lemma 3.3. Equation (1.2) holds if and only if

$$
\sum_{n \geq 0} \mathrm{e}^{a n}\left(W_{n+1}-W_{n}\right) \quad \text { converges a.s. }
$$

Proof of Theorem 1.1. Under the assumptions of the theorem, Lemma 3.2 implies that $\left(M_{n}\right)_{n \in \mathbb{N}_{0}}$ is an $\mathcal{L}^{2}$-bounded martingale, in particular,

$$
\sum_{n \geq 0} \mathrm{e}^{a n}\left(\widetilde{W}_{n+1}-W_{n}+R_{n}\right) \quad \text { converges a.s. }
$$

This is equivalent to the almost-sure convergence of the series $\sum_{n \geq 0} \mathrm{e}^{a n}\left(\widetilde{W}_{n+1}-W_{n}\right)$ since $\sum_{n \geq 0} \mathrm{e}^{a n} R_{n}<\infty$ a.s. in view of (3.11). Another appeal to (3.11) yields

$$
\sum_{n \geq 0} \mathrm{P}\left(\tilde{W}_{n+1} \neq W_{n+1}\right)<\infty
$$


which directly implies that $\mathrm{P}\left(\widetilde{W}_{n+1} \neq W_{n+1}\right.$ infinitely often $)=0$ by an application of the Borel-Cantelli lemma. Hence,

$$
\sum_{n \geq 0} \mathrm{e}^{a n}\left(W_{n+1}-W_{n}\right) \quad \text { converges a.s., }
$$

which is equivalent to (1.2) by Lemma 3.3.

\section{Acknowledgement}

The authors thank an anonymous referee for a careful reading of the paper and for pointing out several oversights of the authors.

\section{References}

[1] Alsmeyer, G. And IKsanov, A. (2009). A log-type moment result for perpetuities and its application to martingales in supercritical branching random walks. Electron. J. Prob. 14, 289-312.

[2] Alsmeyer, G., Iksanov, A., Rösler, U. and Polotsky, S. (2009). Exponential rate of $\mathcal{L}^{p}$-convergence of intrinsic martingales in supercritical branching random walks. Theory Stoch. Process. 15, 1-18.

[3] Asmussen, S. and Hering, H. (1983). Branching Processes (Progress Prob. Statist. 3). Birkhäuser, Boston, MA.

[4] Beljaev, Ju. K. and Maksimov, V. M. (1963). Analytical properties of a generating function for the number of renewals. Theory Prob. Appl. 8, 108-112.

[5] Biggins, J. D. (1977). Martingale convergence in the branching random walk. J. Appl. Prob. 14, 25-37.

[6] Biggins, J. D. And Kyprianou, A. E. (1997). Seneta-Heyde norming in the branching random walk. Ann. Prob. 25, 337-360.

[7] Bingham, N. H., Goldie, C. M. and Teugels, J. L. (1989). Regular Variation (Encyclopaedia Math. Appl. 27). Cambridge University Press.

[8] Borovkov, A. A. (1962). New limit theorems in boundary-value problems for sums of independent terms. Sibirsk. Mat. Ž. 3, 645-694.

[9] Gatzouras, D. (2000). On the lattice case of an almost-sure renewal theorem for branching random walks. Adv. Appl. Prob. 32, 720-737.

[10] Heyde, C. C. (1964). Two probability theorems and their application to some first passage problems. J. Austral. Math. Soc. 4, 214-222.

[11] Heyde, C. C. (1966). Some renewal theorems with application to a first passage problem. Ann. Math. Statist. 37, 699-710.

[12] IKsanov, A. M. (2006). On the rate of convergence of a regular martingale related to a branching random walk. Ukrainian Math. J. 58, 368-387.

[13] LIU, Q. (1997). Sur une équation fonctionnelle et ses applications: une extension du théorème de Kesten-Stigum concernant des processus de branchement. Adv. Appl. Prob. 29, 353-373.

[14] LyONs, R. (1997). A simple path to Biggins' martingale convergence for branching random walk. In Classical and Modern Branching Processes (Minneapolis, MN, 1994; IMA Vol. Math. Appl. 84), Springer, New York, pp. 217-221.

[15] Nerman, O. (1981). On the convergence of supercritical general (C-M-J) branching processes. Z. Wahrscheinlichkeitsth 57, 365-395.

\section{Added in proof}

After the present paper was accepted for publication we learned that parts (b) and (c) of our Theorem 2.1 have been proved in [4] via a more complicated argument. 